CIRJE-F-1123

\title{
Corrigendum to Crawford and Sobel (1982) "Strategic Information Transmission"
}

\author{
Haruki Kono \\ Graduate School of Economics, The University of Tokyo \\ Michihiro Kandori \\ The University of Tokyo \\ August 2019
}

CIRJE Discussion Papers can be downloaded without charge from:

http://www.cirje.e.u-tokyo.ac.jp/research/03research02dp.html

Discussion Papers are a series of manuscripts in their draft form. They are not intended for circulation or distribution except as indicated by the author. For that reason Discussion Papers may not be reproduced or distributed without the written consent of the author. 


\title{
Corrigendum to Crawford and Sobel (1982) "Strategic Information Transmission"
}

\author{
KONO, Haruki ${ }^{2}$
}

KANDORI, Michihiro ${ }^{3}$

August 30, 2019

${ }^{1}$ This note is based on the discussion in the undergraduate research seminar organized by the second author, of which the first author is a member. We thank all the participants and especially Fuhito Kojima, who played a role of guest instructor, for their helpful discussion. We are also extremely grateful to Vincent Crawford and Joel Sobel for their encouragement and valuable suggestions, which greatly improved this note. All remaining errors are ours. The second author acknowledes the financial support form KAKENHI Number JP17K03617.

e-mail: haruki730@gmail.com

${ }^{3}$ e-mail: kandori@e.u-tokyo.ac.jp 


\begin{abstract}
In their analysis of strategic information transmission, Vincent (Crawford and Joel Sobel (1982) showed the existance of partition equilibria (Theorem 1). Although the theorem itself is correct, the proof contains some incorrect statements. We present a counter-example and provide a correct version of the proof.
\end{abstract}




\section{Introduction}

Vincent Crawford and Joel Sobel (11982) discovered a remarkable property that, in their general model of strategic information transmission, if there is a partition equilibrium where the state space $[0,1]$ is partitioned into $N$ intervals, then for any integer $1 \leq n \leq N$, there is also a partition equilibrium with size $n$ (Theorem 1). While this claim is true, the proof is built on some incorrect statements. We point this out by means of a counter-example and propose a correct version of the proof.

Their model consists of a sender $S$ and a receiver $R$. The state of nature $m$ is a random variable with density $f$ on its support $[0,1]$, and $m$ is observed only by the sender. After observing $m$, the sender costlessly sends an arbitrary message to the receiver. The receiver observes the message and takes an action $y \in \mathbb{R}$. The payoffs of the sender and the receiver are $U^{S}(y, m)$ and $U^{R}(y, m)$ respectively. The payoff functions are twice continuously differentiable, and each function is strictly concave in $y\left(U_{11}^{i}<0, i=S, R\right)$ and has a unique maximizer for each $m$ denoted by $y^{i}(m), i=S, R$. It is assumed that the ideal points of the sender and the receiver are distinct; $y^{S}(m) \neq y^{R}(m)$ for all $m$. Moreover, $U_{12}^{i}>0, i=S, R$.

An partition equilibrium is defined to be a perfect Bayesian equilibrium, where (i) the state space $[0,1]$ is partitioned into $N$ intervals $\left[0, a_{1}\right],\left[a_{1}, a_{2}\right], \ldots\left[a_{N-1}, 1\right]$ and (ii) the sender reveals to the receiver which interval contains the realized state $m$. Lemma 1 of the paper correctly establilshes that (i) any equilibrium is a partition equilibrim and (ii) the size of partition $N$ is bounded. After Lemma 1, the paper presents Theorem 1, and its main contents are summarized as follows. Let $\bar{y}\left(a, a^{\prime}\right)$ be the receiver's optimal action when she knows that $m$ lies in $\left[a, a^{\prime}\right]$.

Theorem 1' [1] Partition $\left[0, a_{1}\right],\left[a_{1}, a_{2}\right], \ldots\left[a_{N-1}, 1\right]$ consititutes a partition equilibrium if and only if

$$
\begin{array}{rlrl}
U^{S}\left(\bar{y}\left(a_{i-1}, a_{i}\right), a_{i}\right) & =U^{S}\left(\bar{y}\left(a_{i}, a_{i+1}\right), a_{i}\right) & (i=1,2, \cdots, N-1), \\
a_{0} & =0, & & ((11) \text { in the paper }) \\
a_{N} & =1 . & & ((12) \text { in the paper })
\end{array}
$$

[2] There exists an integer $N(b)>0$ such that a partition equilibrium of every size from one to $N(b)$ exists.

[3] Any equilibrium is equivalent to one in this class. 
Condition (田) is the key to characterize the equilibrium and it is called the arbitrage condition. It states that the sender who is just on the boundary of $\left[a_{i-1}, a_{i}\right]$ and $\left[a_{i}, a_{i+1}\right]$ (i.e., the sender who knows $m=a_{i}$ ) is indifferent between reporting that $m$ is in $\left[a_{i-1}, a_{i}\right]$ and in $\left[a_{i}, a_{i+1}\right]$. Note that (田) defines a second order difference equation.

Parts [1] and [3] are correctly proved (the last paragraph of page 1438 and onwards). ${ }^{\mathbb{W}}$ We found, however, the proof of part [2] depends on incorrect assertions. They define, on page 1438

$$
K(a):=\max \left\{i \mid(\mathbb{A}) \text { has a solution } 0=a_{0}<a_{1}=a<\cdots<a_{i} \leq 1\right\}
$$

and argue that the following is true.

If $K\left(a_{1}\right)=N$ and $K(a)$ is discontinuous at $a=a_{1}$, then $a$ satisfies (11) and (12).

This is a key step to prove statement [2] in their proof. However, we found that this statement can fail when $y^{S}(m)<y^{R}(m)$. More precisely, we found that condition (12) $a_{N}=1$ can fail at the point of discontinuity.

The basic intuition is as follows. The proof correctly observes that, when there is a solution $0=a_{0}<a_{1}<\cdots<a_{N}<1$, in the neighborhood of this solution the terminal point $a_{N}$ varies continuously with respect to the choice of $a_{1}$ (note that the second order difference equation (四) is being solved with given initial conditions $0=a_{0}<a_{1}$ ). The above assertion basically states that, by a suitable choice of $a_{1}$, the terminal point $a_{N}$ can hit 1 , and at this point a solution with the given length $(N+1)$ disappears (this is the discontinuous point of $K(a)$ in (因)). However, this claim is not warranted because as we vary $a_{1}$, the penultimate point $a_{N-1}$ can possibly hit $a_{N}$ before the latter reaches 1 , and at this point $(\mathbb{A})$ can cease to have a solution with the given length $(N+1)$. Indeed, we provide a counter-example in the next section in which this happens. Because of this property, the following main implication of the above assertion to prove [2] also fails in our example;

(因) has a solution $0=a_{0}^{\prime}<a_{1}^{\prime}<\cdots<a_{n}^{\prime}=1$ for any $1 \leq n \leq$ $N(b)$,

${ }^{1} \mathrm{~A}$ minor comment to [1]: Partition equilibrium with size $1,0=a_{0}<a_{1}=1$, does not satisfy (因) because (因) is a second order difference equation and it should be defined on $\left(a_{0}, a_{1}, a_{2}\right)$ (here, $a_{2}$ does not exist). This is a minor semantic issue that does not cause any difficulty in the proof. This statement can be interpreted as the fact that the model always has the 1-partition equilibrium where no information is transmitted.

${ }^{2}$ Page 1438, the fourth line from the bottom. The last part of this sentence should be read as " $a_{1}$ satisfies (11) and (12).". 
where $N(b):=\sup _{a \in(0,1]} K(a)$.

In the end, we will show that those assertions in the proof are in fact correct provided that $y^{R}(m)<y^{S}(m)$, and we provide a correct version of the proof for statement [2] (the first part of Section 3). Then we go on to show how to prove statement [2] when $y^{R}(m)<y^{S}(m)$ is not satisfied.

\section{A Counter-Example}

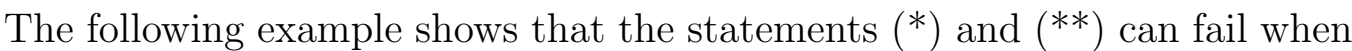
$y^{S}(m)<y^{R}(m)$. In this example, (四) has a solution $0=a_{0}^{\prime}<a_{1}^{\prime}<a_{2}^{\prime}<1$ but it fails to have another solution $0=a_{0}^{\prime \prime}<a_{1}^{\prime \prime}<a_{2}^{\prime \prime}=1$, This shows that their statement (荬) in the previous section is not true, basically because in this example condition (12) $a_{N}=1$ can never be satisfied. Exact proof that this example violates (河) is given at the end of this section. Their assertion (苂) in the previous section also fails, because in this example $N(b):=\sup _{a \in(0,1]} K(a) \geq 2$.

Let us describe our example. The sender's utility is

$$
U^{S}(y, m)=-y^{2}+h(m) y-\frac{1}{10},
$$

where $h(\cdot)$ is strictly increasing and satisfies $h(m)=2 m-\frac{1}{10}$ if $m \leq \frac{2}{5}$, and if $m \geq \frac{2}{5}, h(m)$ only slightly increases from $h\left(\frac{2}{5}\right)=\frac{7}{10}$. More precisely,

$$
h(m)-\frac{7}{10}<\varepsilon \text { if } m \geq \frac{2}{5} .
$$

for some small $\varepsilon>0$. We choose $h$ in such a way that it is twice continuously differentiable. Obviously, such a function $h$ exists. All the assumptions in the paper are satisfied; $U_{11}^{S}<0, U_{12}^{S}>0$, and $U^{S}$ is twice continuously differentiable. We assume $m$ is uniformly distributed over $[0,1]$ and $U^{R}(y, m)=-(y-m)^{2}$.

First we show that the ideal points of the sender and the receiver are distinct; $y^{S}(m) \neq y^{R}(m)$ for all $m$. The first order condition $0=U_{1}^{S}=$ $-2 y+h(m)$ shows $y^{S}(m)=\frac{h(m)}{2}$. Therefore, if $m \leq \frac{2}{5}$,

$$
y^{S}(m)=m-\frac{1}{20}<m=y^{R}(m) .
$$


If $m>\frac{2}{5}, \frac{h(m)}{2}<\frac{7}{2 \times 10}+\frac{\varepsilon}{2}$, and therefore, if $\varepsilon$ is small enough,

$$
y^{S}(m)<\frac{7}{20}+\frac{\varepsilon}{2}<\frac{2}{5}<m=y^{R}(m) .
$$

Thus we conclude that $y^{S}(m)<y^{R}(m)$ for all $m$.

Next, we show that (因) has a solution $0=a_{0}^{\prime}<a_{1}^{\prime}=\frac{2}{5}<a_{2}^{\prime}=\frac{3}{5}<1$. When the state of nature is $m=a=\frac{2}{5}$, the sender's payoff is factorized as

$$
U^{S}\left(y, \frac{2}{5}\right)=-y^{2}+\frac{7}{10} y-\frac{1}{10}=-\left(y-\frac{1}{5}\right)\left(y-\frac{1}{2}\right) .
$$

Note that $\frac{1}{5}$ is the receiver's best reply when she knows $m \in\left[0, \frac{2}{5}\right]$ (denoted $\left.\bar{y}\left(0, \frac{2}{5}\right)\right)$ and that $\frac{1}{2}$ is the receiver's best reply when she knows $m \in\left[\frac{2}{5}, \frac{3}{5}\right]$ (denoted $\bar{y}\left(\frac{2}{5}, \frac{3}{5}\right)$ ). Hence, the above expression shows that (因) is satisfied with $a_{0}=0, a_{1}=\frac{2}{5}, a_{2}=\frac{3}{5}$ (both sides of the equation (因) is zero).

We now show that (因) fails to have another solution $0=a_{0}^{\prime \prime}<a_{1}^{\prime \prime}<a_{2}^{\prime \prime}=$ 1. In particular, we show that the associated condition (A)

$$
U^{S}\left(\bar{y}\left(0, a_{1}\right), a_{1}\right)=U^{S}\left(\bar{y}\left(a_{1}, 1\right), a_{1}\right)
$$

cannot be satisfied with any $a=a_{1}$. In a 2-partition equilibrium, the state space $[0,1]$ is partitioned into $[0, a]$ and $[a, 1]$, and the receiver chooses $\bar{y}(0, a)=\frac{a}{2}$ in the former and $\bar{y}(a, 1)=\frac{a+1}{2}$ in the latter. Therefore, the above condition boils down to

$$
g(a):=U^{S}\left(\frac{a}{2}, a\right)-U^{S}\left(\frac{a+1}{2}, a\right)=0 .
$$

Our functional form of $U^{S}$ implies

$$
\begin{aligned}
g(a) & =\left(-\frac{a^{2}}{4}+h(a) \frac{a}{2}-\frac{1}{10}\right)-\left(-\frac{a^{2}+2 a+1}{4}+h(a) \frac{a+1}{2}-\frac{1}{10}\right) \\
& =\frac{1}{4}+\frac{1}{2} a-\frac{1}{2} h(a) .
\end{aligned}
$$

By plugging in the functional form of $h$, we obtain

$$
g(a)=\frac{3}{10}-\frac{1}{2} a \text { if } a \leq \frac{2}{5}
$$


which is strictly positive (it is minimized $g\left(\frac{2}{5}\right)=\frac{1}{10}>0$ ). For $a>\frac{2}{5}$, we have

$$
\begin{aligned}
g(a) & >\frac{1}{4}+\frac{1}{2} a-\frac{1}{2}\left(\varepsilon+\frac{7}{10}\right) \\
& =\frac{1}{2} a-\frac{1}{10}-\frac{\varepsilon}{2} \\
& >\frac{1}{2} \cdot \frac{2}{5}-\frac{1}{10}-\frac{\varepsilon}{2} \\
& =\frac{1}{10}-\frac{\varepsilon}{2}>0,
\end{aligned}
$$

for small enough $\varepsilon$. We conclude that $g(a)>0$ for all $a$ and therefore there is no $a \in[0,1]$ that satisfies the required condition $g(a)=0$ (condition (四)). Hence, there is no sequence $0=a_{0}^{\prime \prime}<a_{1}^{\prime \prime}<a_{2}^{\prime \prime}=1$ that satisfies (田).

What happens in this example is that, as we vary $a_{1}$, the solution to (因) has the property that the penultimate point $a_{1}$ can hits the terminal point $a_{2}$ before the latter reaches 1 , and at that point (太A) ceases to have a solution with length three $\left(0=a_{0}, a_{1}, a_{2}\right)$. This happens at $a_{1}=a^{*}$ such that $\left(a_{0}, a_{1}, a_{2}\right)=\left(0, a^{*}, a^{*}\right)$, and the existence of $a^{*} \in\left(\frac{2}{5}, 1\right)$ is demonstrated as follows. $a^{*}$ is a solution to

$$
U^{S}(\bar{y}(0, a), a)=U^{S}(\bar{y}(a, a), a) .
$$

Since we consider positive solutions, our definition of $U^{S}$ induces that this equation is equivalent to

$$
h(a)=\frac{3}{2} a .
$$

Consider the following continuous function

$$
\phi(a):=h(a)-\frac{3}{2} a .
$$

Note that $\phi$ is strictly decreasing in $\left(\frac{2}{5}, 1\right)$ because $h^{\prime}(a)$ is almost zero if $\varepsilon$ is small enough, and therefore $\phi^{\prime}(a)=h^{\prime}(a)-\frac{3}{2}<0$. The property of $h$ implies

$$
\phi\left(\frac{2}{5}\right)=h\left(\frac{2}{5}\right)-\frac{3}{2} \cdot \frac{2}{5}=\frac{7}{10}-\frac{3}{5}>0,
$$

and for small $\varepsilon$

$$
\phi(1)=h(1)-\frac{3}{2} \cdot 1<\frac{7}{10}+\varepsilon-\frac{3}{2}<0 .
$$


Therefore, there exists $a^{*} \in\left(\frac{2}{5}, 1\right)$ such that $\phi\left(a^{*}\right)=0$, equivalently

$$
U^{S}\left(\bar{y}\left(0, a^{*}\right), a^{*}\right)=U^{S}\left(\bar{y}\left(a^{*}, a^{*}\right), a^{*}\right) .
$$

Indeed, $a^{*}$ is a counter-example of (因). To show this, we confirm

$$
K\left(a^{*}\right)=1,
$$

$K$ is discontinuous at $a=a^{*}$,

$a^{*} \neq 1$ ((12) does not hold).

Firstly, to prove (ii) we show that there is no $a^{\prime} \in\left(a^{*}, 1\right)$ such that

$$
U^{S}\left(\bar{y}\left(0, a^{*}\right), a^{*}\right)=U^{S}\left(\bar{y}\left(a^{*}, a^{\prime}\right), a^{*}\right) .
$$

Computing this equation, we obtain

$$
a^{\prime}=2\left(h\left(a^{*}\right)-a^{*}\right) .
$$

Recall that $0=\phi\left(a^{*}\right)=h\left(a^{*}\right)-\frac{3}{2} a^{*}$. Then the above condition is written as

$$
a^{\prime}=a^{*} \text {. }
$$

This is not compatible with the assumption that $a^{\prime}$ lies in $\left(a^{*}, 1\right)$. This fact implies that there is no solution to (因) with the initial condition $\left(0=a_{0}, a^{*}\right)$ with the length three or more. Hence, (ii) holds.

To show (囵), we prove the following claim:

$$
\text { for all } a \in\left(\frac{2}{5}, a^{*}\right), K(a) \geq 2 \text {. }
$$

Take $a \in\left(\frac{2}{5}, a^{*}\right)$ and solve

$$
U^{S}(\bar{y}(0, a), a)=U^{S}\left(\bar{y}\left(a, a^{\prime}\right), a\right) .
$$

As we have seen above, we obtain $a^{\prime}=2(h(a)-a)$. Now we show that $a^{\prime} \in(a, 1)$. From the strict monotonicity of $\phi$,

$$
a^{\prime}-a=2 h(a)-3 a=2 \phi(a)>2 \phi\left(a^{*}\right)=0,
$$

and for small $\varepsilon$,

$$
1-a^{\prime}=1-2 h(a)+2 a>1-2 h(a)+2 \cdot \frac{2}{5}=\frac{9}{5}-2 h(a)>\frac{9}{5}-\frac{7}{5}-2 \varepsilon>0
$$

holds. Therefore, $0<a<a^{\prime}(<1)$ is a solution to (田), and consequently,

$$
\liminf _{a \rightarrow a^{*}-0} K(a) \geq 2>1=K\left(a^{*}\right),
$$

which implies (西).

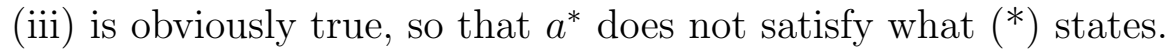




\section{Corrections to the Proof}

Given the correct statements [1] in Theorem 1' and Lemma 1 in the paper (which shows that there is the maximum size $N(b)$ of partition equilibria), it is clear that statement [2] of Theorem 1' is equvalent to the following.

Claim 1. If the second order difference equation (四) has a solution $0=a_{0}^{\prime}<$ $a_{1}^{\prime}<\cdots<a_{N}^{\prime}=1$, then for any integer $2 \leq n \leq N$, it has another solution $0=a_{0}^{\prime \prime}<a_{1}^{\prime \prime}<\cdots<a_{n}^{\prime \prime}=1$.

In this section, we directly prove this Claim to supply the correct proof of statement [2]. Obviously, the above claim is true if the following holds:

For for any integer $n \geq 2$, if the second order difference equation

(田) has a solution $0=a_{0}^{\prime}<a_{1}^{\prime}<\cdots<a_{n}^{\prime}<1$, it has another (\#) solution $0=a_{0}^{\prime \prime}<a_{1}^{\prime \prime}<\cdots<a_{n}^{\prime \prime}=1$.

Vincent ('rawtord and Joel Sobel (148:2) basically tried to prove statement [2] of Theorem 1' by the above assertion, but our counter-example shows that it is not valid if $y^{R}(m)>y^{S}(m)$. We first show that this assertion is in fact true provided that $y^{R}(m)<y^{S}(m)$. Then we go on to show that Claim $\square$ also holds when $y^{R}(m)<y^{S}(m)$ is not satisfied.

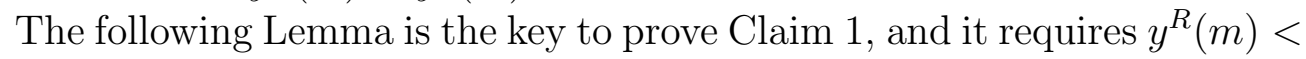
$y^{S}(m)$. This lemma guarantees that, as we vary $a_{1}, a_{n}$ never hits $a_{n+1}$ before the latter reaches 1 ,

Lemma 1. Suppose that $y^{S}(m)>y^{R}(m)$ for all $m \in[0,1]$. Then, there exists $\varepsilon>0$ such that for any partial partition $\left(a_{0}, \cdots, a_{N}\right)$ satisfying (田),

$$
a_{n+1}-a_{n}>\varepsilon \quad(n=1, \cdots, N-1) .
$$

Remark 1. The original proof correctly observes a similar property $a_{n+2}-$ $a_{n}>\varepsilon$ for some $\varepsilon>0$, which holds under the general assumption $y^{S}(m) \neq$ $y^{R}(m)$ for all $m$, but this is not enough to prove $(\mathbb{\#})$, as our counter-example shows.

\footnotetext{
${ }^{3}$ This implies that $K(a)$ jumps down by 1 and condition (12) is satisfied at any discontinuity point, as asserted in the proof of the paper. Hence their statements in the proof are correct when $y^{R}(m)<y^{S}(m)$. Since it is possible to prove Claim $\square$ directly without introducing function $K(a)$, we chose this simpler way to provide a correct version of the proof, rather than closely following their original argument that is given in terms of the properties of function $K(a)$.
} 
Proof. Since $y^{S}$ and $y^{R}$ are strictly increasing, their inverses are well-defined and continuous on compact domain $\left[y^{S}(0), y^{R}(1)\right]$. Therefore, $\min _{y}\left(y^{R^{-1}}(y)-\right.$ $\left.y^{S^{-1}}(y)\right)$ exists, and it is strictly positive because $y^{S}(m)>y^{R}(m)$. We denote this by $\varepsilon$. The arbitrage condition ( $(\mathbb{A})$ states that the sender who knows $m=a_{n}$ has identical payoff at $\bar{y}\left(a_{n-1}, a_{n}\right)$ and $\bar{y}\left(a_{n}, a_{n+1}\right)$, and the concavity of the payoff implies that the peak of the payoff, which is achieved by $y^{S}\left(a_{n}\right)$, is in between those points;

$$
\bar{y}\left(a_{n-1}, a_{n}\right)<y^{S}\left(a_{n}\right)<\bar{y}\left(a_{n}, a_{n+1}\right) .
$$

Moreover, the monotonicity of $\bar{y}$ also induces

$$
\bar{y}\left(a_{n}, a_{n+1}\right)<\bar{y}\left(a_{n+1}, a_{n+1}\right)=y^{R}\left(a_{n+1}\right) .
$$

Thus we have obtained

$$
y^{S}\left(a_{n}\right)<y^{R}\left(a_{n+1}\right) .
$$

From the definition of $\varepsilon$ and the monotonicity of $y^{R^{-1}}$,

$\varepsilon \leq y^{R^{-1}}\left(y^{S}\left(a_{n}\right)\right)-y^{S^{-1}}\left(y^{S}\left(a_{n}\right)\right)<y^{R^{-1}}\left(y^{R}\left(a_{n+1}\right)\right)-y^{S^{-1}}\left(y^{S}\left(a_{n}\right)\right)=a_{n+1}-a_{n}$.

Remark 2. This inequality does not necessarily hold for $n=0$ (i.e., it is not necessarily true that $\left.a_{1}-a_{0}>\varepsilon\right)$ because $a_{0}$ and $a_{1}$ are the initial conditions for the second order difference equation (因) and therefore $a_{0}<a_{1}$ can be chosen arbitrarily.

Next we show the following lemma to formally prove the continuity of the solution to (田) with respect to the initial conditions.

Lemma 2. Let $g(x, y)$ be a continuous function defined on $W \subset \mathbb{R}^{2}$ such that, for each $x, g(x, y)$ is stictly decreasing in $y$. If $g\left(x^{\prime}, y^{\prime}\right)=0$, then there exsit an open neighborhood of $x^{\prime}$ denoted $V \subset\{x \mid(x, y) \in W\}$ and a continuous function $\phi: V \rightarrow \mathbb{R}$ such that $\phi\left(x^{\prime}\right)=y^{\prime}$ and $g(x, \phi(x))=0$ for all $x \in V$.

Remark 3. This is a slight generalization of the implicit function theorem, where the assumption $\partial g / \partial y \neq 0$ in the implicit function theorem is replaced with $g$ being strictly decreasing in $y$. The proof relies on the intermediate value theorem. If we employ an additional assumption that the probability 
density function $f(m)$ is continuous (Vincent Crawford and .oel Sobell (11.982) did not assume this), in what follows we can just use the standard implicit function theorem instead of this lemma because the continuity of $f$ implies the differentiability of $\bar{y}$.

Proof. For any given $\varepsilon>0$, choose $y$ and $\bar{y}$ such that (i) $y<y^{0}<\bar{y}$, (ii) $\left(x^{0}, y\right) \in W$ and $\left(x^{0}, \bar{y}\right) \in W$, and (iii) $\bar{y}-y<\varepsilon$. Since $g$ is strictly decreasing in $y$, we obtain $g\left(x^{0}, y\right)>0$ and $g\left(x^{0}, \bar{y}\right)<0$. Continuity of $g$ implies that there is $\delta>0$ such that $\left|x^{1}-x^{0}\right|<\delta \Rightarrow g\left(x^{1}, \underline{y}\right)>0$ and $g\left(x^{1}, \bar{y}\right)<0$. Continuity of $g$ in $y$ and the intermediate value theorem imples that, when $\left|x^{1}-x^{0}\right|<\delta$, there is $y^{1} \in(y, \bar{y})$ such that $g\left(x^{1}, y^{1}\right)=0$. Given that $g$ is strictly decreasing in $y, y^{1}$ is unique for any given $x^{1}$. Choose any pair of $\varepsilon$ and $\delta$ in the above statement and denote the pair by $(\bar{\varepsilon}, \bar{\delta})$. The above argument shows that, for $V=\left\{x^{1}|| x^{1}-x^{0} \mid<\bar{\delta}\right\}$, there is a unique function $\phi: V \rightarrow \mathbb{R}$ that satisfy $\phi\left(x^{0}\right)=y^{0}$ and $g\left(x^{1}, \phi\left(x^{1}\right)\right)=0$ for all $x^{1} \in V$, where $\phi\left(x^{1}\right)$ is equal to $y^{1}$ in the above argument. Furthermore, $\phi$ is continuous at $x^{0}$ because for any $\varepsilon>0$, there is $\delta>0$ such that $\left|x^{1}-x^{0}\right|<$ $\delta \Rightarrow\left|\phi\left(x^{1}\right)-\phi\left(x^{0}\right)\right|=\left|y^{1}-y^{0}\right|<\varepsilon$. The last inequality holds because $y^{0}, y^{1} \in(y, \bar{y})$ and $\bar{y}-y<\varepsilon$. Applying the same argument for any $x \in V$ shows that $\phi$ is continuous on $V$.

Next, recall that $\bar{y}(a, b)$ is the maximizer of $F(a, b, y):=\int_{a}^{b} U^{R}(y, m) f(m) d m$. Since $F$ is continuous and strictly concave in $y$, Berge's maximum theorem shows that $\bar{y}(a, b)$ is continuous. Inspection of the first order condition shows that $\bar{y}(a, b)$ is strictly increasing in $a$ and $b$.

Now we are ready to formally prove the continuity of the solution of (四) with respect to the initial conditions, which was rather informally alluded in the proof of Theorem 1 in Vincent Crawford and Inel Sobel (1982). This lemma is proved by repeated applications of Lemma 2 .

Lemma 3. If the second order difference equation (田) has a solution $0=$ $a_{0}^{\prime}<a_{1}^{\prime}<\cdots<a_{n}^{\prime}<1$, then there exist

$$
\begin{gathered}
V: \text { an open neighborhood of } a_{1}^{\prime} \text { and } \\
\text { continuous } \phi_{i}: V \rightarrow \mathbb{R} \quad(i=2, \cdots, n)
\end{gathered}
$$

such that

$$
\phi_{i}\left(a_{1}^{\prime}\right)=a_{i}^{\prime}
$$


for all $i$, and

$$
\left(0, a_{1}, \phi_{2}\left(a_{1}\right), \cdots, \phi_{n}\left(a_{1}\right)\right) \text { satisfies (田) }
$$

for all $a_{1} \in V$.

Proof. We employ induction on $i=2, \cdots, n$. First we prove the induction part: if there are continuous functions $\phi_{i-2}\left(a_{1}\right)$ and $\phi_{i-1}\left(a_{1}\right)$ such that $\phi_{i-2}\left(a_{1}^{\prime}\right)=a_{i-2}^{\prime}$ and $\phi_{i-1}\left(a_{1}^{\prime}\right)=a_{i-1}^{\prime}$ defined on an open set $V_{i-1}$, then there is a continuous function $\phi_{i}\left(a_{1}\right)$ defined on an open neighborhood of $a_{1}^{\prime}$ denoted by $V_{i} \subset V_{i-1}$ such that $\phi_{i}\left(a_{1}^{\prime}\right)=a_{i}^{\prime}$ and $\left(\phi_{i-2}\left(a_{1}\right), \phi_{i-1}\left(a_{1}\right), \phi_{i}\left(a_{1}\right)\right)$ satisfies (A) for all $a_{1} \in V_{i}$.

Proof of the induction part: Define

$g\left(a_{1}, a_{i}\right):=U^{S}\left(\bar{y}\left(\phi_{i-2}\left(a_{1}\right), \phi_{i-1}\left(a_{1}\right)\right), \phi_{i-1}\left(a_{1}\right)\right)-U^{S}\left(\bar{y}\left(\phi_{i-i}\left(a_{1}\right), a_{i}\right), \phi_{i-1}\left(a_{1}\right)\right)$.

Note that $g$ is continuous. Furthermore, since $a_{l-2}^{\prime}, a_{l-1}^{\prime}$ and $a_{l}^{\prime}$ satisfy (因),

$$
g\left(a_{1}^{\prime}, a_{i}^{\prime}\right):=U^{S}\left(\bar{y}\left(a_{i-2}^{\prime}, a_{i-1}^{\prime}\right), a_{i-1}^{\prime}\right)-U^{S}\left(\bar{y}\left(a_{i-1}^{\prime}, a_{i}^{\prime}\right), a_{i-1}^{\prime}\right)=0 .
$$

This implies that the peak of strictly concave function $U^{S}\left(\cdot, a_{i-1}^{\prime}\right)$ lies in interval $\left[\bar{y}\left(a_{i-2}^{\prime}, a_{i-1}^{\prime}\right), \bar{y}\left(a_{i-1}^{\prime}, a_{i}^{\prime}\right)\right]$ and therefore $U_{1}^{S}\left(\bar{y}\left(a_{i-1}^{\prime}, a_{i}^{\prime}\right), a_{i-1}^{\prime}\right)<0$ (recall $\left.U_{1}^{S}=\partial U^{S} / \partial y\right)$. Continuity of $U_{1}^{S}$ and the fact that $\bar{y}\left(a_{i-1}, a_{i}\right)$ is continuous and strictly increasing in $a_{i}$ imply that there is a small enough open neighborhood of $\left(a_{1}^{\prime}, a_{i}^{\prime}\right)$ denoted $W \subset V_{i-1} \times \mathbb{R}$ such that $U^{S}\left(\bar{y}\left(\phi_{i-i}\left(a_{1}\right), a_{i}\right), \phi_{i-1}\left(a_{1}\right)\right)$ is strictly decreasing in $a_{i}$ (and therefore so is $g\left(a_{1}, a_{i}\right)$ ) on $W$. Hence all the assumptions in Lemma $\nabla$ are satisfied (if $g$ is viewed as a function defined on $W)$ and the conclusion of the induction part holds.

Next, observe that $\phi_{0}\left(a_{1}\right) \equiv 0$ and $\phi_{1}\left(a_{1}\right)=a_{1}$ are continuous and defined on $V_{1}:=\mathbb{R}$. This shows that the premise of the induction part is true for $i=2$. Thererfore, the conclusion of the induction part holds for all $i=2, \cdots, n$, and the lemma follows for $V=V_{n}$.

Now we are ready to state our key result.

Theorem 1. Statement $(\mathbb{\#})$ and therefore Claim $\square$ hold if $y^{S}(m)>y^{R}(m)$ for all $m$.

Proof. Assume that $(\mathbb{\nabla})$ has a solution $0=a_{0}^{\prime}<a_{1}^{\prime}<\cdots<a_{n}^{\prime}<1$. Let

$$
D:=\left\{a_{1} \in(0,1): \text { there exists } 0<a_{1}<\cdots<a_{n} \leq 1 \text { satisfying }(\mathbb{\mathrm { A }})\right\} .
$$


Our assumption implies that $D$ is not empty. Let $a_{1}^{\prime \prime}:=\sup D$. Then, there exists a sequence in $D$ converging to $a_{1}^{\prime \prime}$. We denote this by $\left\{a_{1}^{(i)}\right\}_{i \in \mathbb{N}}$. Also, we denote the solution to (田) associated with $a_{1}^{(i)}$ by $\left(0, a_{1}^{(i)}, a_{2}^{(i)}, \cdots, a_{n}^{(i)}\right)$. Since the sequence $\left\{\left(a_{1}^{(i)}, a_{2}^{(i)}, \cdots, a_{n}^{(i)}\right)\right\}_{i \in \mathbb{N}}$ lies in a compact set $[0,1]^{n}$, there exists a convergent subsequence $\left\{\left(a_{1}^{\left(i_{k}\right)}, a_{2}^{\left(i_{k}\right)}, \cdots, a_{n}^{\left(i_{k}\right)}\right)\right\}_{k \in \mathbb{N}}$ that converges to a certain point $\left(a_{1}^{\prime \prime}, a_{2}^{\prime \prime}, \cdots, a_{n}^{\prime \prime}\right)$. By definition, the subsequence satisfies (因);

$$
U^{S}\left(\bar{y}\left(a_{l-1}^{\left(i_{k}\right)}, a_{l}^{\left(i_{k}\right)}\right), a_{l}^{\left(i_{k}\right)}\right)=U^{S}\left(\bar{y}\left(a_{l}^{\left(i_{k}\right)}, a_{l+1}^{\left(i_{k}\right)}\right), a_{l}^{\left(i_{k}\right)}\right) .
$$

By letting $k \rightarrow \infty$, we get

$$
U^{S}\left(\bar{y}\left(a_{l-1}^{\prime \prime}, a_{l}^{\prime \prime}\right), a_{l}^{\prime \prime}\right)=U^{S}\left(\bar{y}\left(a_{l}^{\prime \prime}, a_{l+1}^{\prime \prime}\right), a_{l}^{\prime \prime}\right)
$$

beaches $U^{S}$ and $\bar{y}$ are continuous. Therefore, $\left(0=a_{0}^{\prime \prime}, a_{1}^{\prime \prime}, \cdots, a_{n}^{\prime \prime}\right)$ satisfies (四). Our assumption $0<a_{1}$ and $a_{1} \in D$ imply $a_{1}^{\prime \prime}>0$ because $a_{1}^{\prime \prime}=\sup D \geq$ $a_{1}>0$. In addition, Lemma $\square$ shows that $a_{1}^{\prime \prime}, a_{2}^{\prime \prime}, \cdots, a_{n}^{\prime \prime}$ are all distinct. Thus, we have obtained $0=a_{0}^{\prime \prime}<a_{1}^{\prime \prime}<a_{2}^{\prime \prime}<\cdots<a_{n}^{\prime \prime}$ that satisfies (四). Lastly, we show $a_{n}^{\prime \prime}=1$. If not, Lemma 3 shows that there exists an open neighborhood $V$ of $a_{1}^{\prime \prime}$ such that each $a_{1}^{0} \in V$ (in particular, some $a_{1}^{0}>a_{1}^{\prime \prime}$ ) lies in $D$. This contradicts the fact that $a_{1}^{\prime \prime}=\sup D$. Therefore, (田) has another solution $0=a_{0}^{\prime \prime}<a_{1}^{\prime \prime}<\cdots<a_{n}^{\prime \prime}=1$.

The above result implies that Theorem 1 in the paper holds when $y^{S}(m)>$ $y^{R}(m)$ for all $m$. Now we show that Claim $\square$ and therefore Theorem 1 are also true in general. When the reverse inequality $y^{S}(m)<y^{R}(m)$ holds for all $m$, we can employ the symmetric argument by solving the second order difference equation (田) "from the top" with the initial conditions $a_{N-1}<a_{N}=1$ to prove Theorem 1. Namely, the symmetric argument shows the following sufficient condition to prove Claim W when $y^{S}(m)<y^{R}(m)$;

For any integer $n \geq 2$, if the second order difference equation (A)

has a solution $0<a_{1}^{\prime}<\cdots<a_{n-1}^{\prime}<a_{n}^{\prime}=1$ it has another solution (\#\#) $0=a_{1}^{\prime \prime}<\cdots<a_{n-1}^{\prime \prime}<a_{n}^{\prime \prime}=1$.

Lastly, we note that there is no other cases under the maintained assumption $y^{S}(m) \neq y^{R}(m)$ for all $m$. This is because if there are $m^{\prime}$ and $m^{\prime \prime}$ such that $y^{S}\left(m^{\prime}\right)>y^{R}\left(m^{\prime}\right)$ and $y^{S}\left(m^{\prime \prime}\right)<y^{R}\left(m^{\prime \prime}\right)$, then the continuity of $y^{S}(\cdot)$ and $y^{R}(\cdot)$ imply that there is $m^{0}$ such that $y^{S}\left(m^{0}\right)=y^{R}\left(m^{0}\right)$, which contradicts the maintained assumption. Hence, we have shown that statement [2] of Theorem 1' and therefore Theorem 1 in Vincent Crawford and Inel Sobel (1982) are true. 


\section{Reference}

V. P. Crawford and J. Sobel, "Strategic Information Transmission," Econometrica: Journal of the Econometric Society, pp. 1431-1451, 1982. 